\title{
Successful Management of Poorly Controlled Type 2 Diabetes with Multidisciplinary Neurobehavioral Rehabilitation: A Case Report and Review
}

\author{
Zhihui Deng (D) · John Davis · Flor Muniz-Rodriguez • \\ Fran Richardson
}

Received: April 4, 2018 / Published online: May 28, 2018

(C) The Author(s) 2018

\begin{abstract}
Lifestyle modification with healthy diet and physical exercise is considered the basic strategy of prevention and treatment of type 2 diabetes, a commonly seen comorbidity in patients with acquired brain injury. Additionally, emotional stress with anxiety and depression is suggested to play a role in type 2 diabetes. Research studies have demonstrated the efficacy of multidisciplinary lifestyle intervention in patients with inadequate glycemic control. However, whether lifestyle approaches alone may be adequate for the management of poorly controlled type 2 diabetes is unknown. We report a 30 -year-old male patient whose type 2 diabetes was inadequately controlled by 50 units of insulin glargine, 15 units of insulin aspart supplement with meals plus a correctional scale as well as multiple oral hypoglycemic drugs when
\end{abstract}

Enhanced digital features To view enhanced digital features for this article go to https://doi.org/10.6084/ m9.figshare.6275948.

Z. Deng $(\varangle) \cdot$ F. Muniz-Rodriguez

Division of Physical Medicine and Rehabilitation, Department of Medicine, McMaster University,

Hamilton, Canada

e-mail: dengz22@mcmaster.ca

Z. Deng · J. Davis · F. Muniz-Rodriguez ·

F. Richardson

Hamilton Health Sciences Regional Rehabilitation

Centre, Hamilton, Canada admitted to a neurobehavioral rehabilitation unit subsequent to his brain injury. Following 3 months of multidisciplinary rehabilitation for his functional neurological symptom disorder, all his pharmacological agents were gradually discontinued and his diabetes was successfully managed solely by lifestyle approaches.

Keywords: Lifestyle intervention; Neurobehavioral rehabilitation; Physical exercise; Type 2 diabetes mellitus

\section{INTRODUCTION}

The World Health Organization (WHO) reported 422 million diabetes patients globally in 2014 and diabetes will be the seventh leading cause of death in 2030. Diabetes is a major cause of blindness, kidney failure, heart attacks, stroke, and lower limb amputation. Type 2 diabetes accounts for more than 90\% of all diabetes cases in the world with an increased prevalence in developed countries due to obesity, physical inactivity, and the growing population of older age individuals [1]. Healthy diet, regular physical activity, and maintaining a normal body weight are believed to prevent or delay the onset of type 2 diabetes. These approaches are also beneficial for patients with type 2 diabetes [2, 3]. Recent research has demonstrated the particular importance of 
intensive lifestyle intervention in patients with inadequate glycemic control [4]. However, successful glycemic control by lifestyle intervention alone is rarely seen in patients whose diabetes is poorly controlled by medications. We present one successful case that demonstrates a significantly beneficial effect of physical exercise and lifestyle intervention in the management of type 2 diabetes mellitus.

\section{CASE DESCRIPTION}

A 30-year-old man was admitted to a neurobehavioral rehabilitation unit as a result of the development of a functional neurological symptom disorder following a concussion sustained in a motor vehicle accident 3 years earlier. The patient had a history of type 2 diabetes for 8 years, but managed this successfully with only physical activities and no medication prior to the accident. Unfortunately, there is no available data of his glucose values or HbA1C level prior to his injury. Following the accident, he developed depression, anxiety with the features of post-traumatic stress disorder, and functional neurological symptoms with gait and speech dysfunction, with brain imaging studies showing no intracranial pathology. Further complicating the clinical presentation were chronic pain, insomnia, and neurocognitive impairments. Together, these impairments and symptoms prevented the patient from engaging in his normal routine of activities of daily living (ADL) and work. At the time of the admission, he had gained $14 \mathrm{~kg}$ (BMI 28.7) and required 50 units insulin glargine subcutaneous at bedtime, 15 units of insulin aspart three times a day with meals, as well as a correctional scale. Furthermore, he took canagliflozin $300 \mathrm{mg}$ daily and metformin/sitagliptin 50/1000 1 tablet twice a day. In addition, he took rosuvastatin $10 \mathrm{mg}$ daily and fenofibrate $160 \mathrm{mg}$ daily for dyslipidemia.

Psychological assessment included standardized measures (Symptom Checklist 90 Revised, Beck Depression Inventory Revised, Beck Anxiety Inventory, Insomnia Severity Index, Brief Pain Inventory). Findings were valid and showed abnormal elevations related to phobic anxiety, worry about somatic complaints and cognitive impairments, and significant problems with sleep and pain.

In addition to medical care, the patient received transdisciplinary rehabilitation from a team including rehabilitation therapist, occupational therapist, physiotherapist, speech-language pathologist, behavior therapist, and neuropsychologist. Rehabilitation was carried out in a therapeutic milieu in which functional activities were presented individually and in groups, both on the unit and in the community. Activities were organized in a daily schedule that interspersed rehabilitation with rest throughout the day, with both staff and patient recording completion of activities. Activities included physical exercise, as well as personal care, social and recreational pursuits, chores to care for his ADL apartment on the unit, and community-based tasks such as shopping and volunteering. Duration and intensity of activities were gradually increased during the course of admission, with goals adjusted collaboratively.

Psychological treatment in the form of cognitive behavior therapy was directed to reducing phobic avoidance of proximity to motor vehicles. As the patient progressed successfully through a hierarchy of phobic situations, his mobility increased from using a walker to running. Relaxation and mindfulness exercises were also conducted, and self-directed practice introduced. Relaxation skills were integrated with sleep hygiene methods. Psychoeducational intervention was provided related to the expected process of return to health following concussion.

With the application of multidisciplinary approaches on the neurobehavioral rehabilitation unit, he gradually made functional gains during a 4-month admission. Psychometric findings from readministration of standardized measures shortly before discharge showed a resolution of anxiety, worry, sleeping difficulty, and pain. Physically he discontinued the usage of a rollator walker for ambulating and started aerobic exercises and resistance training while his mood and behavior were improving. Approximately 3 months after his admission, all his hypoglycemic agents, including insulin and 
oral medications, had been gradually weaned off, and he had lost more than $10 \mathrm{~kg}$ (BMI 25.0). His HbA1C dropped from $10.5 \%$ to $6.4 \%$ and his blood glucose was well controlled without any pharmacological agents (capillary blood glucose (CBG) varied between 4.0 and $8.0 \mathrm{mmol} / \mathrm{L}$ ). Meanwhile, rosuvastatin and fenofibrate were discontinued as his triglyceride reduced from 4.15 to $0.89 \mathrm{mmol} / \mathrm{L}$, and nonHDL cholesterol from 3.50 to $3.16 \mathrm{mmol} / \mathrm{L}$.

\section{Compliance with Ethics Guidelines}

The authors received consent from the patient included in this case report.

\section{DISCUSSION}

Type 2 diabetes is a common metabolic disorder that affects more than one in every 10 adults in the USA and Canada who are 20 years or older, and is associated with an increased risk of coronary heart disease and microvascular diseases including retinopathy, nephropathy, and peripheral neuropathy. Diabetes is also among the common comorbidities in patients admitted to the setting where the patient was seen, i.e., a neurobehavioral rehabilitation unit in this case. Multiple medications are often required for glycemic control. Poorly controlled diabetes is a frustrating but commonly seen phenomena for clinical health providers. Besides pharmacological intervention, there is growing evidence of the effectiveness of lifestyle modification on glycemic control in patients with type 2 diabetes.

Clinical trials have demonstrated that physical exercise is an effective strategy for the management of type 2 diabetes. Both aerobic and resistance training are beneficial for the management of blood glucose, lipids, and blood pressure and thus reduce cardiovascular complication and mortality $[5,6]$. According to the position statements published by the American College of Sports Medicine, the American Diabetes Association, and the American Heart Association, lifestyle intervention with regular exercises should be the first-line strategy for the prevention and control of type 2 diabetes $[7,8]$.
Supervised structured training was shown to be more effective for HbA1C reduction than unstructured exercise, so participating in a welldesigned exercise program is recommended for type 2 diabetes patients [9].

Following a motor vehicle accident, the patient in this report developed significant behavioral disturbance in the form of functional neurological symptoms, stopped all physical exercise, and experienced the worsening of his diabetes. Upon admission to the neurobehavioral rehabilitation unit, he presented with a significant balance impairment and ambulated with the aid of a rollator walker. With structured and supportive physical and behavioral rehabilitation, his abnormal gait resolved, and he gradually made functional gains seen in his successful self-management of his ADL on the rehabilitation unit and in the community, as well as completion of a healthy exercise routine with a combination of aerobic and resistance training. After 3 months of rehabilitation he no longer took medication while continuing to participate in routine physical activity, including daily community walking for $30 \mathrm{~min}$ at a time, running $1.5 \mathrm{~km}$ 3-4 times per week, and daily gym exercises for strength training with rotating muscle groups. Meanwhile, he played leisure sports such as basketball. Progressing with physical gains was accompanied by a gradual improvement of his glycemic control.

In addition to exercises, dietary approach has been suggested to play an essential role in the lifestyle intervention for patients with type 2 diabetes [10]. Following the motor vehicle accident, this patient developed a pattern of poor sleep habits characterized by often sleeping during the day and staying up through the night. At the same time, his eating schedule was highly inconsistent and he did not observe the diet restrictions for diabetes. During his stay on the neurobehavioral rehabilitation unit, he ate meals at regular times and established healthy sleep hygiene. Diabetic diet was provided under the supervision of the registered dietitian (1600-1800 calories per day with $15-20 \%$ protein and 50\% carbohydrates plus diet drink/ dessert). He was compliant with diabetic diet during his rehabilitation. These lifestyle 
changes worked synergistically with physical training contributing to his weight loss and better glycemic control.

There is evidence that different types of emotional stress, including depression and anxiety, are associated with an increased risk for the development of type 2 diabetes $[11,12]$. The mechanism underlying this could be associated with unhealthy lifestyle behaviors, chronic activation of hypothalamic-pituitary-adrenal axis and sympathetic nervous system, and immune system dysfunction. Psychological intervention has been shown to be effective in the management of type 2 diabetes $[13,14]$. Our patient developed significant mood and behavioral disturbance following the motor vehicle accident. Multidisciplinary neurobehavioral rehabilitation with psychological intervention led to a resolution of his anxiety, worry, sleeping difficulty, and pain. The effective management of his emotional issues was likely a factor contributing to the successful control of his diabetes.

Previous research studies have demonstrated the efficacy of multidisciplinary approach in patients with poorly controlled type 2 diabetes $[3,4]$. This patient was likewise managed with the aforementioned intensive lifestyle approaches combined with the support of a multidisciplinary rehabilitation team. The success of this case provides evidence for the importance and benefit of multidisciplinary lifestyle intervention in inadequately controlled type 2 diabetes. However, with only one case, we cannot identify the group of type 2 diabetes patients for whom lifestyle and behavioral intervention might be sufficient treatment.

Nevertheless, compliance with medical advice is a significant issue in patients with type 2 diabetes, and it is challenging in the real world for patients to pursue lifestyle modification without support. Diet modification and intense physical training without professional supervision could be detrimental for patients with poorly controlled type 2 diabetes, especially if they take statins and fibrates for dyslipidemia. Short-term rehabilitation with supportive service from a multidisciplinary team can be beneficial for those patients. However, no patient will be able to stay in such a costly and comprehensive program for long. Ongoing support for these diabetic patients to maintain their functional gains is an important public health and socioeconomic problem. In this case, the patient self-reported his maintenance of lifestyle changes 6 months after discharge, successful return to work, continuing follow-up by his family physician, and no further use of medication to treat diabetes.

There may be factors other than neurobehavioral approaches responsible for his good clinical outcome. It is unclear whether there was any error in his injection techniques considering his high dose of daily insulin use prior to the admission to our rehabilitation unit, although this was less likely as a family member who is a registered pharmacist was overseeing and assisting. Therefore, we cannot conclude with certainty that the loss of body weight coupled with neurobehavioral interventions was the sole cause for the successful control of his diabetes.

\section{CONCLUSION}

Though lifestyle modification is a well-known approach for preventing and managing type 2 diabetes, the effectiveness of lifestyle approaches may not be fully understood by clinical physicians, care providers, or patients. In the case reported here, a combination of dietary advice, regular aerobic exercise and resistance training, as well as multidisciplinary neurobehavioral intervention was organized and systematically implemented in a highly structured approach to providing rehabilitation for a complex group of comorbidities, with highly beneficial results. The remarkable outcome of his poorly controlled type 2 diabetes occurred incidentally with the rehabilitation of a complex set of comorbidities that followed a significant personal injury which had disrupted his normal lifestyle. This case provides striking evidence of the potential benefit of lifestyle intervention in the management of this disease. While acknowledging the scientific limitations of this case presentation, the patient's success likely resulted from the synergistic effects of the multiple aforementioned interventions. 
Although it is not clear which components of his rehabilitation were essential, the present results support the use of intensive lifestyle intervention for patients with inadequately controlled type 2 diabetes in a structured rehabilitation program. Ongoing support for these patients to maintain their functional gains remains an important public health issue.

\section{ACKNOWLEDGEMENTS}

We thank the patient in the study and all the team members who provided care for him on the neurobehavioral rehabilitation unit. We are grateful to Dr. Shanker Nesathurai for his comments on an earlier version of the manuscript.

Funding. No funding was received for this study or the publication of this report.

Authorship. All named authors meet the International Committee of Medical Journal Editors (ICMJE) criteria for authorship of this manuscript, take responsibility for the integrity of the work as a whole, and have given their final approval of the version to be published.

Disclosures. Zhihui Deng, John Davis, Flor Muniz-Rodrigues, Fran Richardson have no conflicts to disclose.

Compliance with Ethics Guidelines. The authors received consent from the patient included in this case report.

Open Access. This article is distributed under the terms of the Creative Commons Attribution-NonCommercial 4.0 International License (http://creativecommons.org/licenses/ by-nc/4.0/), which permits any noncommercial use, distribution, and reproduction in any medium, provided you give appropriate credit to the original author(s) and the source, provide a link to the Creative Commons license, and indicate if changes were made.

\section{REFERENCES}

1. Deshpande AD, Harris-Hayes M, Schootman M. Epidemiology of diabetes and diabetes-related complications. Phys Ther. 2008;88(11):1254-64.

2. Johansen MY, MacDonald CS, Hansen KB, et al. Effect of an intensive lifestyle intervention on glycemic control in patients with type 2 diabetes: a randomized clinical trial. JAMA. 2017;318(7):637-46.

3. Maislos M, Weisman D. Multidisciplinary approach to patients with poorly controlled type 2 diabetes mellitus: a prospective, randomized study. Acta Diabetol. 2004;41(2):44-8.

4. Sbroma Tomaro E, Pippi R, Reqinato E, et al. Intensive lifestyle intervention is particularly advantageous in poorly controlled type 2 diabetes. Nutr Metab Cardiovasc Dis. 2017;27(8):688-94.

5. De Feo P, Schwarz P. Is physical exercise a core therapeutical element for most patients with type 2 diabetes? Diabetes Care. 2013;36(Suppl 2):S149-54.

6. Umpierre D, Ribeiro PA, Kramer CK, et al. Physical activity advice only or structured exercise training and association with HbA1c levels in type 2 diabetes: a systematic review and meta-analysis. JAMA. 2011;305(17):1790-9.

7. Colberg SR, Siqal RJ, Fernhall B, et al. Exercise and type 2 diabetes: the American College of Sports Medicine and the American Diabetes Association: joint position statement executive summary. Diabetes Care. 2010;33(12):2692-6.

8. Marwick TH, Hordern MD, Miller T, et al. Exercise training for type 2 diabetes mellitus: impact on cardiovascular risk: a scientific statement from the American Heart Association. Circulation. 2009;119(25):3244-62.

9. Sanghani NB, Parchwani DN, Palandurkar KM, et al. Impact of lifestyle modification on glycemic control in patients with type 2 diabetes mellitus. Indian J Endocrinol Metab. 2013;17(6):1030-9.

10. Vetter ML, Amaro A, Volger S. Nutritional management of type 2 diabetes mellitus and obesity and pharmacologic therapies to facilitate weight loss. Postgrad Med. 2014;126(1):139-52.

11. Hackett RA, Steptoe A. Type 2 diabetes mellitus and psychological stress-a modifiable risk factor. Nat Rev Endocrinol. 2017;13(9):547-60. 
12. Pouwer F, Kupper N, Adriaanse MC. Does emotional stress cause type 2 diabetes mellitus? A review from the European Depression in Diabetes (EDID) Research Consortium. Discov Med. 2010;9(45):112-8.

13. Chapman A, Liu S, Merkouris S, et al. Psychological interventions for the management of glycemic and psychological outcomes of type 2 diabetes mellitus in China: a systematic review and meta-analyses of randomized controlled trials. Front Public Health. 2015;3:252.

14. Sudhir PM. Advances in psychological interventions for lifestyle disorders: overview of interventions in cardiovascular disorder and type 2 diabetes mellitus. Curr Opin Psychiatry. 2017;30(5):346-51. 